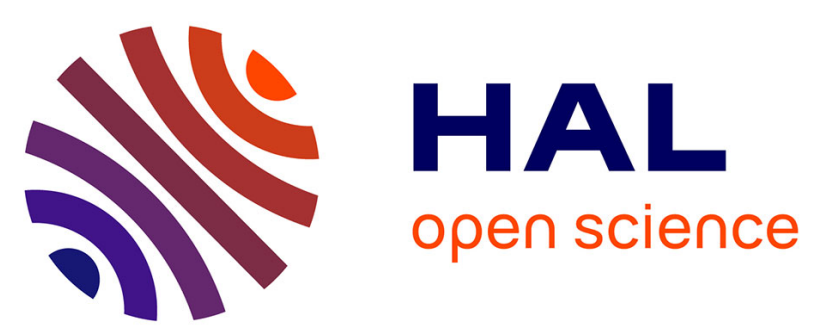

\title{
Boundary port Hamiltonian control of a class of nanotweezers.
}

Héctor Ramirez Estay, Yann Le Gorrec

\section{To cite this version:}

Héctor Ramirez Estay, Yann Le Gorrec. Boundary port Hamiltonian control of a class of nanotweezers.. The European Control Conference, ECC'13., Jan 2013, Switzerland. pp.1-6. hal-00872207

\section{HAL Id: hal-00872207 https://hal.science/hal-00872207}

Submitted on 11 Oct 2013

HAL is a multi-disciplinary open access archive for the deposit and dissemination of scientific research documents, whether they are published or not. The documents may come from teaching and research institutions in France or abroad, or from public or private research centers.
L'archive ouverte pluridisciplinaire HAL, est destinée au dépôt et à la diffusion de documents scientifiques de niveau recherche, publiés ou non, émanant des établissements d'enseignement et de recherche français ou étrangers, des laboratoires publics ou privés. 


\title{
Boundary port Hamiltonian control of a class of nanotweezers
}

\author{
Hector Ramirez and Yann Le Gorrec
}

\begin{abstract}
Boundary controlled-port Hamiltonian systems have proven to be of great use for the analysis and control of a large class of systems described by partial differential equations. The use of semi-group theory, combined with the underlying physics of Hamiltonian systems permits to prove existence, well-possessedness and stability of solutions using constructive techniques. On other hand, the differential geometric representation of these systems has lead to finite dimension approximation methods that conserves physical properties such as the interconnection structure and the energy. These results are applied to the modelling and control of a class of nanotweezers used for DNA-manipulation. The Nanotweezer may be modelled as a flexible beam interconnected with a finite dimensional dynamical system representing the manipulated object. A boundary controlled-port Hamiltonian model for the ensemble and an exponentially stabilizing controller are proposed. A geometric approximation scheme is used to reduce the infinite dimensional system and numerical simulations of the closed-loop system presented.
\end{abstract}
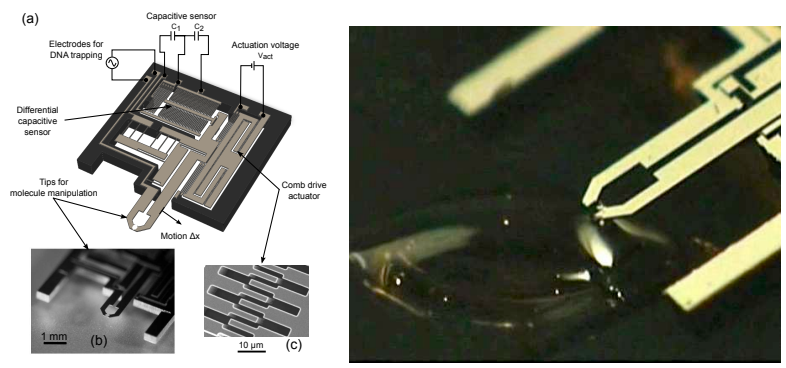

Fig. 1. Silicon nanotweezers

\section{INTRODUCTION}

Recent technological progresses have made possible the manipulation of single biological molecules by using several devices or methods such as: magnetic tweezers [1], [2], optical tweezers [3], AFM cantilevers [4] and microfibers [5], [6]. The single molecule manipulation is of great interest in order to elucidate their basic characteristics and is of particular interest in the case of DNA molecules [7]. Some promising tools for the manipulation of DNA molecules are silicon nanotweezers, (Figure 1).

The principle of silicon nanotweezers is to trap DNA bundle between the two arm tips by using dielectrophoresis and to characterize the DNA mechanical properties by using electrostatic actuation. Such actuator has been used for the monitoring of enzymatic reactions on DNA. It has

This work was supported by French ANR sponsored project HAMECMOPSYS under Reference Code ANR-11-BS03-0002.

Hector Ramirez and Yann Le Gorrec are with the department of Automation and Micro-Mechatronic Systems, FEMTO-ST UMR CNRS 6174, ENSMM, 26 chemin de l'épitaphe, F-25030 Besançon, France. \{ramirez, legorrec\}@femto-st. fr been shown that nanotweezers are so sensitive to the DNA stiffness variation that it becomes flexible. As a consequence, current microfabrication processes tend to reduce the thickness of the beams in order to improve the sensitivity of the actuator. This naturally leads to control problems that may be formulated in the frame of Boundary Control Systems [8]. The work proposed in this paper has been done in the perspective of very compliant actuators.

In this paper we propose to use the framework of port Hamiltonian system for the modelling, control and simulation of a class of nanotweezers. Indeed, this framework is particularly adapted for the case of interconnected systems with some interesting perspectives for the generalization to the manipulation in liquid phase (multiphysic modelling). Furthermore recent results on the stabilization of boundary control systems by using dynamic boundary port Hamiltonian controllers have been given in [9], [10]. These results are recalled and applied to the system under study. The results are validated by numerical simulations of a discretized model of the closed-loop system. The model approximation is performed by using a geometric discretization method [11], which preserves the geometric structure and energetic properties of the boundary controlled-port Hamiltonian model. The paper is organized as follows. In Section II the port Hamiltonian model of the nanotweezer is presented. The model consist of the interconnection of a flexible beam, modelled as a Timoshenko beam, and a DNA-bundle, modelled as a mass-spring-damper system. In Section III the stability analysis of the ensemble twezeer - DNA bundle - controller is analysed using the framework of boundary controlled systems. Section IV presents the geometric reduction of the closed-loop system and some numerical simulations of the displacement of the DNA-bundle. Finally some closing remarks are given in Section $\mathrm{V}$

\section{Port Hamiltonian MOdelling of NANOTWEezers}

A simplified model of a nanotweezer used for DNA manipulation [12] is presented in Figure 2. The trapped DNA bundle is approximated by a mass spring system attached at the tip of the tweezer. The arm is actuated by using electrostatic forces generated by a comb drive actuator. In this paper we do not represent the shuttle and suspension system and consider that we can directly control the force and the torque at the point $a$. We also assume that it is only possible to measure the transversal and angular velocities at the point $a$. The total system, may be divided into three subsystems (Figure 3) the flexible arm, the DNA-bundle at the tip of the gripper and the port Hamiltonian controller. The flexible arm is modelled as a Timoshenko beam (infinite di- 


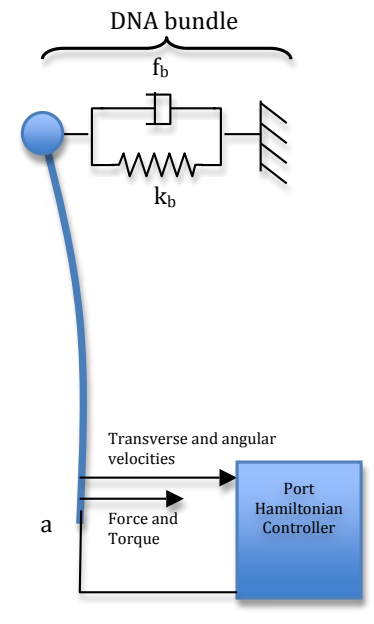

Fig. 2. DNA manipulation through port Hamiltonian control

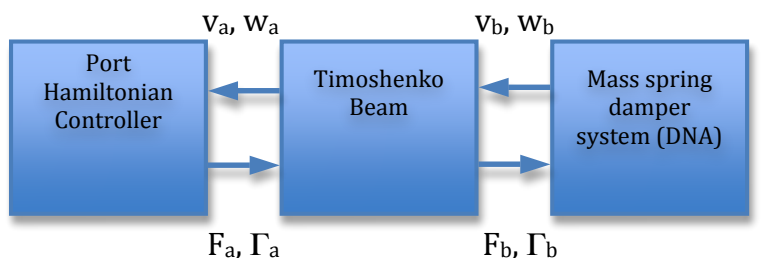

Fig. 3. Interconnexion and control strategy

mensional system) while the DNA-bundle may be modelled as a finite dimensional mechanical system. The subsystems are interconnected through their boundary power conjugated port variables. In Figure 3 the interconnection boundary port variables as well as the causality (depicted with arrows) are given .

\section{A. The Timoshenko beam}

The Timoshenko beam has been widely studied as a distributed parameter port Hamiltonian system [13] and as BCS [14] and the exponential stability of the system has been proved for static boundary feedback [15], [16]. The BCS is defined as

$$
\begin{gathered}
\frac{\partial}{\partial t}\left[\begin{array}{l}
x_{1} \\
x_{2} \\
x_{3} \\
x_{4}
\end{array}\right]=\underbrace{\left[\begin{array}{llll}
0 & 1 & 0 & 0 \\
1 & 0 & 0 & 0 \\
0 & 0 & 0 & 1 \\
0 & 0 & 1 & 0
\end{array}\right]}_{P_{1}} \frac{\partial}{\partial z}\left[\begin{array}{c}
K x_{1} \\
\frac{1}{\rho} x_{2} \\
E I x_{3} \\
\frac{1}{I_{\rho}} x_{4}
\end{array}\right] \\
+\underbrace{\left[\begin{array}{cccc}
0 & 0 & 0 & -1 \\
0 & 0 & 0 & 0 \\
0 & 0 & 0 & 0 \\
1 & 0 & 0 & 0
\end{array}\right]}_{P_{0}}\left[\begin{array}{c}
K x_{1} \\
\frac{1}{\rho} x_{2} \\
E I x_{3} \\
\frac{1}{I_{\rho}} x_{4}
\end{array}\right]
\end{gathered}
$$

where the following state (energy) variables have been defined: $x_{1}=\frac{\partial w}{\partial z}(z, t)-\phi(z, t)$ the shear displacement, $x_{2}=\rho(z) \frac{\partial w}{\partial t}(z, t)$ the transverse momentum distribution, $x_{3}=\frac{\partial \phi}{\partial z}(z, t)$ the angular displacement, and $x_{4}=I_{\rho} \frac{\partial \phi}{\partial t}(z, t)$ the angular momentum distribution, for $z \in(a, b), t \geq 0$, where $w(t, z)$ is the transverse displacement of the beam and $\phi(t, z)$ is the rotation angle of a filament of the beam. The coefficients $\rho(z), I_{\rho}(z), E(z), I(z)$ and $K(z)$ are the mass per unit length, the rotary moment of inertia of a cross section, Youngs modulus of elasticity, the moment of inertia of a cross section, and the shear modulus respectively. The matrices $P_{1}$ and $P_{0}$ defines the skew-symmetric differential operator of order 1 acting on the state space $X=L_{2}\left(a, b, \mathbb{R}^{4}\right), \mathcal{J}=P_{1} \frac{\partial}{\partial z}+P_{0}$. The energy of the beam is expressed in terms of the energy variables,

$$
\begin{aligned}
E & =\frac{1}{2} \int_{a}^{b}\left(K x_{1}^{2}+\frac{1}{\rho} x_{2}^{2}+E I x_{3}^{2}+\frac{1}{I_{\rho}} x_{4}^{2}\right) d z \\
& =\frac{1}{2} \int_{a}^{b} x(z)^{\top}(\mathcal{L} x)(z) d z=\frac{1}{2}\|x\|_{\mathcal{L}}^{2}
\end{aligned}
$$

The boundary port variables are obtained by using integration by parts and factorization in order to define an extended Dirac structure including the boundary [14]. They also can be directly parametrized from $P_{1}$ [14], [15] leading to:

$$
\left[\begin{array}{c}
f_{\partial, \mathcal{L} x} \\
e_{\partial, \mathcal{L} x}
\end{array}\right]=\left[\begin{array}{c}
\left(\rho^{-1} x_{2}\right)(b)-\left(\rho^{-1} x_{2}\right)(a) \\
\left(K x_{1}\right)(b)-\left(K x_{1}\right)(a) \\
\left(I_{\rho}^{-1} x_{4}\right)(b)-\left(I_{\rho}^{-1} x_{4}\right)(a) \\
\left(E I x_{3}\right)(b)-\left(E I x_{3}\right)(a) \\
\left(\rho^{-1} x_{2}\right)(b)+\left(\rho^{-1} x_{2}\right)(a) \\
\left(K x_{1}\right)(b)+\left(K x_{1}\right)(a) \\
\left(I_{\rho}^{-1} x_{4}\right)(b)+\left(I_{\rho}^{-1} x_{4}\right)(a) \\
\left(E I x_{3}\right)(b)+\left(E I x_{3}\right)(a)
\end{array}\right] .
$$

The control objective is to control the translational and angular position of the DNA-bundle. The physical ports are given by the translational force acting at the base of the beam (input), and the translational velocity at the base of the beam (output). All physical ports are hence located on the point $a$ of the beam and directly associated with the dynamic of the suspension mechanism and/or base of the beam. In order to achieve that the input and output variables of the flexible arm coincide with the physical ones we define the following input and outputs for the beam:

$$
\begin{aligned}
& u=\left[\begin{array}{llll}
v(b) & \omega(b) & F(a) & T(a)
\end{array}\right], \\
& y=\left[\begin{array}{llll}
F(b) & T(b) & -v(a) & -\omega(a)
\end{array}\right],
\end{aligned}
$$

which is achieved by defining

$$
u=W\left[\begin{array}{l}
f_{\partial, \mathcal{L} x} \\
e_{\partial, \mathcal{L} x}
\end{array}\right], \quad y=\tilde{W}\left[\begin{array}{l}
f_{\partial, \mathcal{L} x} \\
e_{\partial, \mathcal{L} x}
\end{array}\right],
$$

where

$$
\begin{aligned}
W & =\left[\begin{array}{cccccccc}
1 & 0 & 0 & 0 & 0 & 1 & 0 & 0 \\
0 & 0 & 1 & 0 & 0 & 0 & 0 & 1 \\
0 & -1 & 0 & 0 & 1 & 0 & 0 & 0 \\
0 & 0 & 0 & -1 & 0 & 0 & 1 & 0
\end{array}\right], \\
\tilde{W} & =\left[\begin{array}{ccccccccc}
0 & 1 & 0 & 0 & 1 & 0 & 0 & 0 \\
0 & 0 & 0 & 1 & 0 & 0 & 1 & 0 \\
1 & 0 & 0 & 0 & 0 & -1 & 0 & 0 \\
0 & 0 & 1 & 0 & 0 & 0 & 0 & -1
\end{array}\right] .
\end{aligned}
$$


It can by shown that with this choice of input and output the system (1) defines a an abstract boundary control system. Furthermore $\mathcal{A} x=P_{1}(\partial / \partial z)(\mathcal{L} x)+P_{0} \mathcal{L} x$ with domain

$$
D(\mathcal{A})=\left\{\mathcal{L} x \in H^{1}\left(a, b ; \mathbb{R}^{n}\right) \mid\left[\begin{array}{l}
f_{\partial, \mathcal{L} x}(t) \\
e_{\partial, \mathcal{L} x}(t)
\end{array}\right] \in \operatorname{ker} W\right\}
$$

generates a contraction semigroup on $X$ and the energy balance equation is defined as:

$$
\frac{d E}{d t}=u^{T} y
$$

\section{B. DNA-bundle}

The DNA-bundle may be modelled as ideal mass-springdamper systems, and thus admits a port Hamiltonian system representation. Let us denote with the sub-index $b$ the system representing the DNA-bundle. Then we may write

$$
\begin{aligned}
& \dot{v}_{b}=\left(J_{b}-R_{b}\right) \frac{d E_{b}}{d v_{b}}+g_{b} u_{b} \\
& y_{b}=g_{b}^{\top} \frac{d E_{b}}{d v_{b}},
\end{aligned}
$$

where $v_{b}=\left[q_{b_{1}}, q_{b_{2}}, p_{b_{1}}, p_{b_{2}}\right]^{\top}, q_{b_{1}}, q_{b_{2}}$ are the generalized coordinates, with $q_{b_{1}}$ the distance from the equilibrium configuration and $q_{b_{2}}$ the rotation angle, $p_{b_{1}}, p_{b_{2}}$ are the transversal and rotational generalized momenta respectively, $J_{b}=-J_{b}^{\top}, R_{b}=R_{b}^{\top}>0 \in \mathbb{R}^{4} \times \mathbb{R}^{4}$, the interconnection and damping matrices respectively, defined as

$$
J_{b}=\left[\begin{array}{cc}
0 & I \\
-I & 0
\end{array}\right], \quad R_{b}=\left[\begin{array}{cc}
0 & 0 \\
0 & C_{b}
\end{array}\right]
$$

with $C_{b}=\left[\begin{array}{cc}c_{b_{1}} & 0 \\ 0 & c_{b_{2}}\end{array}\right] \in \mathbb{R}^{2} \times \mathbb{R}^{2}$, where $c_{b_{1}}, c_{b_{2}} \in \mathbb{R}$ are the scalar damping coefficients corresponding to the transversal and rotational translation respectively. The Hamiltonian of the system is given by the kinetic and elastic energy:

$$
E_{b}=\frac{1}{2}\left(k_{b_{1}} q_{b_{1}}^{2}+k_{b_{2}} q_{b_{2}}^{2}\right)+\frac{1}{2}\left(\frac{p_{b_{1}}^{2}}{m_{b}}+\frac{p_{b_{2}}^{2}}{m_{I_{b}}}\right)
$$

where $k_{b_{1}}, k_{b_{2}}$ are the translational and rotational spring coefficients respectively and $m_{b}, m_{I_{b}}$ are the mass and moment of inertia respectively. The total force acting on the DNA bundle, is completed with the contribution of the transversal and angular force at point $b$, of the beam. Hence the input map is $g_{b} \in \mathbb{R}^{4} \times \mathbb{R}^{2}, g_{b}=\left[\begin{array}{ll}0 & I\end{array}\right]^{\top}$ and the inputs $u_{b}=\left[u_{b_{1}}, u_{b_{2}}\right]^{\top} \in \mathbb{R}^{2}$ may be identified with the boundary variables of the beam at the point $b$

$$
u_{b}=\left[\begin{array}{l}
u_{b_{1}} \\
u_{b_{2}}
\end{array}\right]=\left[\begin{array}{l}
\frac{\partial E}{\partial x_{2}}(b) \\
\frac{\partial E}{\partial x_{4}}(b)
\end{array}\right]=\left[\begin{array}{l}
F(b) \\
T(b)
\end{array}\right] .
$$

The outputs correspond to the transversal and angular velocity of the mass at the point $b$, and as it has seen it corresponds to the inputs at the point $b$, for the flexible arm. The finite dimensional PHS of the DNA-bundle is given by

$$
\begin{aligned}
\dot{v}_{b} & =\left[\begin{array}{cc}
0 & I \\
-I & -C_{b}
\end{array}\right] \frac{d E_{b}}{d v}+\left[\begin{array}{ll}
0 & 0 \\
I & 0
\end{array}\right] u_{b} \\
y_{b} & =\left[\begin{array}{ll}
0 & I
\end{array}\right] \frac{d E_{b}}{d v}
\end{aligned}
$$

and corresponds to a strictly passive system with quadratic dissipation rate $s=\frac{d E_{b}}{d v_{b}}{ }^{\top} C_{b} \frac{d E_{b}}{d v_{b}}$.

\section{Stability ANALYSIS AND CONTROL DESIGN}

In this section we just recall some stability results of dynamic boundary control systems. In [15] it is shown that a power conserving interconnection, i.e.,

$$
\begin{aligned}
& u=r-y_{c}, \\
& y=u_{c},
\end{aligned}
$$

with $r \in \mathbb{R}^{n}$ the new input of the system, of a impedance energy preserving BCS, i.e., that satisfies $\frac{1}{2} \frac{d}{d t}\|x(t)\|_{\mathcal{L}}^{2}=$ $u(t) y(t)$, and a linear strictly positive real (SPR) finite dimensional system defines again a BCS on an extended space. This property is detailed in Theorem 1 .

Theorem 1. [15] Let the state of the open-loop BCS satisfy $\frac{1}{2} \frac{d}{d t}\|x(t)\|_{\mathcal{L}}^{2}=u(t) y(t)$. Consider a LTI strictly passive finite dimensional system with storage function $E_{c}(t)=$ $\frac{1}{2}\left\langle v(t), Q_{c} v(t)\right\rangle_{\mathbb{R}^{m}}, Q_{c}=Q_{c}^{\top}>0 \in \mathbb{R}^{m} \times \mathbb{R}^{m}$. Then the feedback interconnection of the BCS and the finite dimensional system is again a BCS on the extended state space $\tilde{x} \in \tilde{X}=X \times V$ with inner product $\left\langle\tilde{x}_{1}, \tilde{x}_{2}\right\rangle_{\tilde{X}}=\left\langle x_{1}, x_{2}\right\rangle_{\mathcal{L}}+$ $\left\langle v_{1}, Q_{c} v_{2}\right\rangle_{V}$. Furthermore, the operator $\mathcal{A}_{e}$ defined by

$$
\mathcal{A}_{e} \tilde{x}=\left[\begin{array}{cc}
\mathcal{J} \mathcal{L} & 0 \\
B_{c} \mathcal{C} & A_{c}
\end{array}\right]\left[\begin{array}{l}
x \\
v
\end{array}\right]
$$

with

$$
\begin{gathered}
D\left(\mathcal{A}_{e}\right)= \\
\left\{\left[\begin{array}{l}
x \\
v
\end{array}\right] \in\left[\begin{array}{l}
X \\
V
\end{array}\right] \mid \mathcal{L} x \in H^{N}\left(a, b ; \mathbb{R}^{n}\right),\left[\begin{array}{c}
f_{\partial, \mathcal{L} x} \\
e_{\partial, \mathcal{L} x} \\
v
\end{array}\right] \in \operatorname{ker} \tilde{W}_{D}\right\},
\end{gathered}
$$

where

$$
\left.\tilde{W}_{D}=\left[\begin{array}{ll}
W+D_{c} \tilde{W} & C_{c}
\end{array}\right)\right]
$$

generates a contraction semigroup on $\tilde{X}$.

In [15] it is shown that asymptotic stability of closed loop system can be proved as long as the finite dimensional system is a positive system. It has also been proved that in the case of static feedback exponential stability can be achieved if the feedback is strictly positive [16]. These results have recently been extended and in [9], [10] it has been shown that these results can be generalized to strictly passive controllers.

Theorem 2. [9], [10] Consider the BCS defined by Theorem 1 with $r(t)=0$, for all $t \geq 0$. If the linear finite dimensional control system is strictly passive, then the BCS system is exponentially stable.

Now, for the ensemble nanotweezer - DNA bundle controller, the control strategy consists in applying a constant force $F_{a}^{*}$ to drive the system to the desired equilibrium configuration $q_{b}^{*}$. The control is completed with a transversal and angular velocity feedback loop in the point $a$ in order to stabilize the system around some equilibrium $q_{b}^{*}$. The problem is similar to the one solved in [10], [13] using energy shaping methods, but we will exploit the port Hamiltonian structure of the global system to define a BCS and use Theorem 2 to guarantee exponential stability. 
It remains to consider a controller at the point $a$. To this end a simple static velocity feedback loop is considered

$$
y_{1}^{a}=-k_{1} v(a), \quad y_{2}^{a}=-k_{2} \omega(a) .
$$

where $k_{1}, k_{2}>0$ are the control gains. This feedback introduces dissipation at the point $a$ and is the most simples case of interconnection of dissipative system. Hence the dissipation of the complete extended system is given by $s=$ $\frac{d E_{a}}{d v_{a}} C_{a} \frac{d E_{a}}{d v_{a}}-k_{1}{\frac{\partial E}{\partial x_{1}}}^{2}(a)-k_{2} \frac{\partial^{2}}{\partial x_{3}}(a)$ implying specifically that we can increase the damping of the system.

\section{SPATIAL REDUCTION AND SIMULATION}

Sections II and III present abstract formulations for the construction of the mathematical model of the system and the synthesis of the controller. In this section we exploit the port Hamiltonian structure of the abstract control system to reduce it to an explicit finite dimensional port Hamiltonian system, which can be simulated using standard numerical algorithms. To this end the mixed-finite element discretization method proposed in [11] is used. The method is based in approximating flows and efforts with differential forms related to their physical (geometrical) natural. In the case of the Timoshenko beam, defined on a one-dimensional spatial domain, we distinguish between zero forms (functions), corresponding to the efforts (force and torque) and one-forms, corresponding to the flows (translational and angular velocities). The reader is referred to [11], [17]-[20] for detailed revisions of the method, and to [21] for its application to the flexible beam.

\section{A. Power preserving discretization}

The infinitesimal energy variables (flow variables) are denoted by $f^{x_{i}}, i=1, \ldots, 4$, and corresponds to the partial derivative with respect to time of the state variables. Their approximation on an infinitesimal section $\mathcal{L}_{a b}$ of an one dimensional spatial domain $\mathcal{L}$ is given by

$$
f^{x_{i}}(t, z)=f_{a b}^{x_{i}} \omega_{a b}^{x_{i}}(z)
$$

where the one-forms $\omega_{a b}^{x_{i}}$ satisfy

$$
\int_{\mathcal{L}_{a b}} \omega_{a b}^{x_{i}}=1
$$

The co-energy variables (infinitesimal efforts) are denoted by $e^{x_{i}}$ and corresponds to the variational derivative of the Hamiltonian (energy). Their approximation on $\mathcal{L}_{a b}$ is given by

$$
e^{x_{i}}(t, z)=e_{a}^{x_{i}} \omega_{a}^{x_{i}}(z)+e_{b}^{x_{i}} \omega_{b}^{x_{i}}(z),
$$

where the zero-forms $\omega_{a}^{x_{i}}, \omega_{b}^{x_{i}}$, satisfy

$$
\omega_{a}(a)^{x_{i}}=1, \omega_{a}(b)^{x_{i}}=0, \omega_{b}(a)^{x_{i}}=0, \omega_{b}(b)^{x_{i}}=1 .
$$

The dynamic equations of the flexible structure may then be approximated by replacing (4) and (5) in (1) as follows

$$
\begin{aligned}
& f_{a b}^{x_{1}} \omega_{a b}^{x_{1}}=e_{a}^{x_{2}} d \omega_{a}^{x_{1}}+e_{b}^{x_{2}} d \omega_{b}^{x_{2}}-e_{a}^{x_{4}} \omega_{a}^{x_{4}} d z-e_{b}^{x_{4}} \omega_{b}^{x_{4}} d z \\
& f_{a b}^{x_{2}} \omega_{a b}^{x_{2}}=e_{a}^{x_{1}} d \omega_{a}^{x_{1}}+e_{b}^{x_{1}} d \omega_{b}^{x_{1}} \\
& f_{a b}^{x_{3}} \omega_{a b}^{x_{3}}=e_{a}^{x_{4}} d \omega_{a}^{x_{4}}+e_{b}^{x_{4}} \omega_{b}^{x_{4}} \\
& f_{a b}^{x_{4}} \omega_{a b}^{x_{4}}=e_{a}^{x_{3}} d \omega_{a}^{x_{3}}+e_{b}^{x_{3}} d \omega_{b}^{x_{3}}+e_{a}^{x_{1}} \omega_{a}^{x_{1}} d z+e_{b}^{x_{1}} \omega_{b}^{x_{1}} d z
\end{aligned}
$$

where $d$ denotes the exterior derivative of differential forms [22]. From the previous relation the following compatibility conditions between one and zero forms are deduced

$$
\begin{aligned}
-\omega_{a b}^{x_{i}} & =d \omega_{a}^{x_{i}} & \omega_{a b}^{x_{i}} & =d \omega_{b}^{x_{i}} \\
\int_{\mathcal{L}_{a b}} \omega_{a}^{x_{4}} d z \omega_{a b}^{x_{1}} & =\omega_{a}^{x_{4}} d z & \int_{\mathcal{L}_{a b}} \omega_{b}^{x_{4}} d z \omega_{a b}^{x_{1}} & =\omega_{b}^{x_{4}} d z \\
\int_{\mathcal{L}_{a b}} \omega_{a}^{x_{1}} d z \omega_{a b}^{x_{4}} & =\omega_{a}^{x_{1}} d z & \int_{\mathcal{L}_{a b}} \omega_{b}^{x_{1}} d z \omega_{a b}^{x_{4}} & =\omega_{b}^{x_{1}} d z
\end{aligned}
$$

Using the compatibility conditions an algebraic relation between the approximated flows and efforts is obtained

$$
\begin{aligned}
f_{a b}^{x_{1}} & =e_{b}^{x_{2}}-e_{a}^{x_{2}}-\int_{\mathcal{L}_{a b}} \omega_{a}^{x_{4}} d z e_{a}^{x_{4}}-\int_{\mathcal{L}_{a b}} \omega_{b}^{x_{4}} d z e_{b}^{x_{4}} \\
f_{a b}^{x_{2}} & =e_{b}^{x_{1}}-e_{a}^{x_{1}} \\
f_{a b}^{x_{3}} & =e_{b}^{x_{4}}-e_{a}^{x_{4}} \\
f_{a b}^{x_{4}} & =e_{b}^{x_{1}}-e_{a}^{x_{1}}+\int_{\mathcal{L}_{a b}} \omega_{a}^{x_{1}} d z e_{a}^{x_{1}}+\int_{\mathcal{L}_{a b}} \omega_{b}^{x_{1}} d z e_{b}^{x_{1}}
\end{aligned}
$$

Since the goal is to achieve a finite dimensional port Hamiltonian system it is necessary to guarantee that the approximated system in energy preserving. To this end the net power of the infinitesimal section is calculated $P_{a b}^{\text {net }}=\sum_{i=1}^{4} \int_{\mathcal{L}_{a b}} f^{x_{i}} e^{x_{i}}+f^{B} e^{B}$, where $f^{B} e^{B}$ is the energy flowing through the boundaries of the system. To identify the port-variables of the discretized interconnection structure $P_{a b}^{\text {net }}$ is expressed in terms of the approximated flows and efforts, and using the compatibility conditions we obtain (6)

$$
P_{a b}^{\text {net }}=\sum_{i=1}^{4} f_{a b}^{x_{i}} e_{a}^{x_{i}} b+f^{B} e^{B}
$$

where

$$
\begin{array}{ll}
e_{a b}^{x_{1}}=\alpha^{\prime} e_{a}^{x_{1}}+\alpha e_{b}^{x_{1}}, & e_{a b}^{x_{2}}=\alpha e_{a}^{x_{2}}+\alpha^{\prime} e_{b}^{x_{2}} \\
e_{a b}^{x_{3}}=\alpha^{\prime} e_{a}^{x_{3}}+\alpha e_{b}^{x_{3}}, & e_{a b}^{x_{4}}=\alpha e_{a}^{x_{4}}+\alpha e_{b}^{x_{4}}
\end{array}
$$

correspond to the generalized efforts, with $\alpha \in[0,1], \alpha=$ $\int_{\mathcal{L}_{a b}} \omega_{b}^{x_{1}}(z) \omega_{a b}^{x_{1}}(z), \alpha^{\prime}=1-\alpha$ and where $f^{B} e^{B}=e_{b}^{x_{1}} e_{b}^{x_{3}}+$ $e_{b}^{x_{2}} e_{b}^{x_{4}}-e_{a}^{x_{1}} e_{b}^{x_{3}}-e_{a}^{x_{2}} e_{a}^{x_{4}}$. The generalized efforts (9) are the conjugated efforts of the approximated flow variables, and permit to express the net power (8) as the scalar pairing

$$
P_{a b}^{\text {net }}=\left\langle e_{a b} \mid f_{a b}\right\rangle=\sum_{i=1}^{4} f_{a b}^{x_{i}} e_{a b}^{x_{i}}+f^{B} e^{B}
$$

where $f_{a b}=\left[f_{a b}^{x_{1}}, f_{a b}^{x_{2}}, f_{a b}^{x_{3}}, f_{a b}^{x_{4}}, f^{B}\right]$ and $e_{a b}=$ $\left[e_{a b}^{x_{1}}, e_{a b}^{x_{2}}, e_{a b}^{x_{3}}, e_{a b}^{x_{4}}, e^{B}\right]$. Hence from (7) and (9) the following 
implicit system is obtained

$$
\begin{aligned}
& \underbrace{\left[\begin{array}{cccccccc}
0 & 0 & 0 & 0 & 0 & 0 & 0 & 0 \\
0 & 0 & 0 & 0 & -\alpha & \alpha^{\prime} & 0 & 0 \\
0 & 0 & 0 & 0 & 0 & 0 & 0 & 0 \\
0 & 0 & 0 & 0 & 0 & 0 & -\alpha & \alpha^{\prime} \\
-1 & 0 & 0 & 0 & 1 & 1 & \beta & -\beta^{\prime} \\
0 & -1 & 0 & 0 & 0 & 0 & 1 & 1 \\
0 & 0 & -1 & 0 & 0 & 0 & 1 & 1 \\
0 & 0 & 0 & -1 & 0 & 0 & 0 & 0
\end{array}\right]}_{F_{a b}}\left[\begin{array}{c}
f_{a b}^{x_{1}} \\
f_{a b}^{x_{2}} \\
f_{a b}^{x_{3}} \\
f_{a b}^{x_{4}} \\
-e_{a}^{x_{2}} \\
e_{b}^{x_{2}} \\
-e_{a}^{x_{4}} \\
e_{b}^{x_{4}}
\end{array}\right]+ \\
& \underbrace{\left[\begin{array}{cccccccc}
-1 & 0 & 0 & 0 & \alpha^{\prime} & \alpha & 0 & 0 \\
0 & -1 & 0 & 0 & 0 & 0 & 0 & 0 \\
0 & 0 & -1 & 0 & 0 & 0 & \alpha^{\prime} & \alpha \\
0 & 0 & 0 & -1 & 0 & 0 & 0 & 0 \\
0 & 0 & 0 & 0 & 0 & 0 & 0 & 0 \\
0 & 0 & 0 & 0 & -1 & 1 & 0 & 0 \\
0 & 0 & 0 & 0 & 0 & 0 & 0 & 0 \\
0 & 0 & 0 & 0 & \beta & \beta^{\prime} & -1 & 1
\end{array}\right]}_{E_{a b}}\left[\begin{array}{l}
e_{a b}^{x_{1}} \\
e_{a b}^{x_{2}} \\
e_{a b}^{x_{3}} \\
e_{a b}^{x_{4}} \\
e_{a}^{x_{1}} \\
e_{b}^{x_{1}} \\
e_{a}^{x_{3}} \\
e_{b}^{x_{3}}
\end{array}\right]=0
\end{aligned}
$$

where $\beta \in[0, b-a], \beta=\int_{\mathcal{L}_{a b}} \omega_{a}^{x_{2}}$ and $\beta^{\prime}=(b-a)-\beta$. The implicit system (10) actually defines a Dirac structure [23], thus implying that the discretized interconnection structure of the infinitesimal section of the Timoshenko beam is power preserving. For simulation and control design purposes it may be convenient to have an explicit representation of (10). This is easily achieved by properly assigning the input and the outputs at the boundaries. In particular, the choice of inputs and outputs (2) leads to the following port Hamiltonian system

$$
\begin{gathered}
{\left[\begin{array}{l}
\dot{x}_{1}^{a b} \\
\dot{x}_{2}^{a b} \\
\dot{x}_{3}^{a b} \\
\dot{x}_{4}^{a b}
\end{array}\right]=\left[\begin{array}{cccc}
0 & -\frac{1}{\alpha} & 0 & -\frac{\beta}{\alpha} \\
\frac{1}{\alpha} & 0 & 0 & 0 \\
0 & 0 & 0 & -\frac{1}{\alpha} \\
\frac{\beta}{\alpha} & 0 & \frac{1}{\alpha} & 0
\end{array}\right]\left[\begin{array}{l}
\frac{\partial E_{a b}}{\partial x_{1}^{a b}} \\
\frac{\partial E_{a b}}{\partial x_{2}^{a b}} \\
\frac{\partial E_{a b}}{\partial x_{3}^{a b}} \\
\frac{\partial E_{a b}}{\partial x_{4}^{a b}}
\end{array}\right]+} \\
{\left[\begin{array}{l}
y_{1}^{a b} \\
y_{2}^{a b} \\
y_{3}^{a b} \\
y_{4}^{a b}
\end{array}\right]=\left[\begin{array}{cccc}
0 & 0 & \frac{1}{\alpha} & 0 \\
-\frac{1}{\alpha} & 0 & 0 & 0 \\
0 & 0 & 0 & \frac{1}{\alpha} \\
0 & -\frac{1}{\alpha} & 0 & 0
\end{array}\right]\left[\begin{array}{l}
u_{1}^{a b} \\
u_{2}^{a b} \\
u_{3}^{a b} \\
u_{4}^{a b}
\end{array}\right],} \\
{\left[\begin{array}{cccc}
0 & -\frac{1}{\alpha} & 0 & 0 \\
0 & 0 & 0 & -\frac{1}{\alpha} \\
\frac{1}{\alpha} & 0 & 0 & 0 \\
0 & 0 & \frac{1}{\alpha} & 0
\end{array}\right]\left[\begin{array}{l}
\frac{\partial E_{a b}}{\partial x_{1}^{a b}} \\
\frac{\partial E_{a b}}{\partial x_{2}^{a b}} \\
\frac{\partial E_{a b}}{\partial x_{3}^{a b}} \\
\frac{\partial E_{a b}}{\partial x_{4}^{a b}}
\end{array}\right]}
\end{gathered}
$$

where $\dot{x}_{i}^{a b}, E_{a b}, u_{i}^{a b}$ and $y_{i}^{a b}$ correspond respectively, to the discretized state variables, Hamiltonian function, input and output port variables on the infinitesimal section $\mathcal{L}_{a b}$. In this case the zero forms have been chosen such that $\alpha=1\left(\alpha^{\prime}=\right.$ $0)$ and $\beta=b-a\left(\beta^{\prime}=0\right)$.

To obtain the complete model of the beam, the infinitesimal sections are interconnected in a power preserving manner. The efforts of the section $j$ that represent outputs in $b$ are interconnected with the efforts of $j+1$ that represent

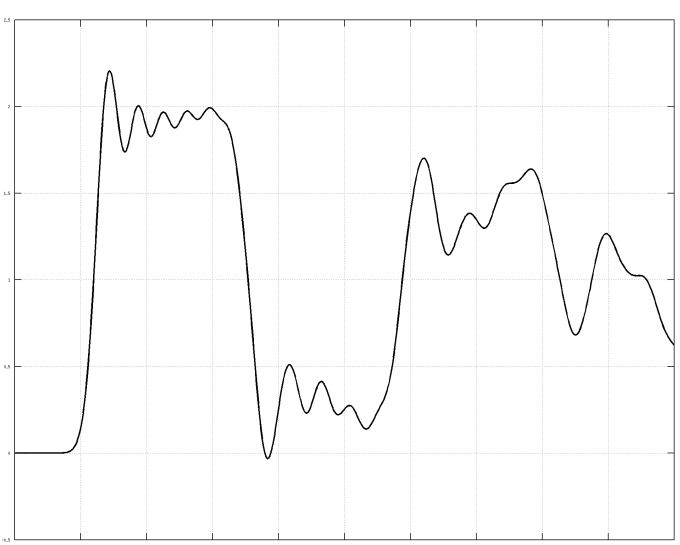

Fig. 4. Open-loop response, $k_{1}=k_{2}=0$.

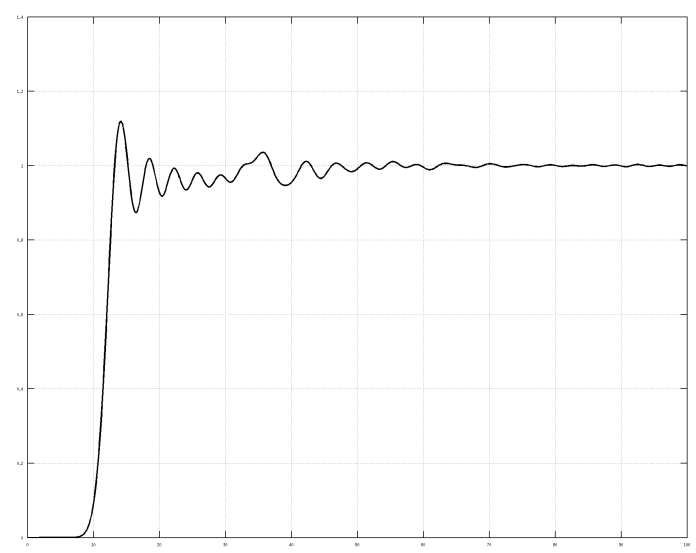

Fig. 5. Closed-loop response, $k_{1}=k_{2}=1$.

the inputs in $a$, and the same for the physical flows. In this case the interconnection of the infinitesimal sections are performed as $u_{1}^{a b}(j+1)=y_{3}^{a b}(j), u_{2}^{a b}(j+1)=y_{4}^{a b}(j)$, $u_{3}^{a b}(j)=-y_{1}^{a b}(j+1)$ and $u_{4}^{a b}(j)=y_{3}^{a b}(j+1)$.

\section{B. Simulations}

Figures 4 and 5 show the transversal position of the DNA-bundle when a step input of force at time $t=0$ is applied at the base of the nanotwezeer. All the numerical parameters of the Timoshenko beam and the DNA-bundle have been selected equal to one $(=1)$ in the simulations, the Timoshenko beam has been divided in 10 infinitesimal subsections, $\mathcal{L}=1$, and $b-a=0.1$. From Figure 4 (openloop response) it may be observed that the system is always exponentially stable since the DNA-bundle acts as strictly passive controller (Theorem 2). However, the static feedback at the point $a$ introduces additional damping to the system and, as expected, the closed-loop system reaches the desired equilibrium considerably faster as may be observed in Figure 5 . 


\section{FINAL REMARKS}

The port Hamiltonian framework has been used to model, control, reduce and simulate a class of nanotweezers used for DNA-manipulation. The ensemble nanotweezer - DNA bundle - controller has been formulated as an abstract control system, namely a boundary controlled port Hamiltonian system and using semi-group theory it has then been shown that the closed-loop system is exponentially stable. The infinite dimensional system has then been approximated as a finite dimensional port Hamiltonian system by using a power preserving discretization scheme that preserves the geometric structure and the energy of the system. Using the approximated model numerical simulations have been carried out to illustrate the results.

The main contribution of this work is to show how the port Hamiltonian framework may be used for practical modelling and control of complex physical systems, in particular a class of nanotweezers for DNA manipulation. We started with an abstract formulation and ended with numerical simulation conserving in every step the physical properties of the system. Future work will deal with the practical implementation of the control scheme using the experimental set-up presented in Figure 1, and with more complex submodels for the DNA-bundle and the controller.

\section{REFERENCES}

[1] F. Amblard, B. Yurke, A. Pargellis, and S. Leibler, "A magnetic manipulator for studying local rheology and micromechanical properties of biological systems," Review of Scientific Instruments, vol. 67, no. 3, pp. 818-827, 1996.

[2] C. Gosse and V. Croquette, "Magnetic tweezers: micromanipulation and force measurement at the molecular level," Biophysical Journal, vol. 82, no. 6, pp. 3314-3329, 2002.

[3] R. Simmons, J. Finer, S. Chu, and J. Spudich, "Quantitative measurements of force and displacement using an optical trap," Biophysical Journal, vol. 70, no. 4, pp. 1813-1822, 1996.

[4] E. Florin, V. Moy, and H. Gaub, "Adhesion forces between individual ligand-receptor pairs," Science, vol. 264, no. 5157, p. 415, 1994.

[5] A. Ishijima, T. Doi, K. Sakurada, and T. Yanagida, "Sub-piconewton force fluctuations of actomyosin in vitro," Nature, 1991.

[6] P. Cluzel, A. Lebrun, C. Heller, R. Lavery, J. Viovy, D. Chatenay, and F. Caron, "Dna: an extensible molecule," Science, vol. 271, no. 5250, p. $792,1996$.

[7] C. Bustamante, Z. Bryant, and S. Smith, "Ten years of tension: singlemolecule dna mechanics," Nature, vol. 421, no. 6921, pp. 423-427, 2003.

[8] R. Curtain and H. Zwart, An introduction to infinite-dimensional linear systems theory, ser. Texts in applied mathematics. New York, USA: Springer-Verlag, 1995

[9] H. Ramirez and Y. L. Gorrec, "Exponential stability of a class of pdes with dynamic boundary control," in Submitted to the American Control Conference, Washington, DC, June 17 - 192013.

[10] A. Macchelli, "Boundary energy shaping of linear distributed portHamiltonian systems," in Proceedings of the 4th IFAC workshop on Lagrangian and Hamiltonian methods for non-linear control, Bertinoro, Italy, August 2012.

[11] G. Golo, V. Talasila, A. van der Schaft, and B. Maschke, "Hamiltonian discretization of boundary control systems," Automatica, vol. 40, no. 5, pp. 757 - 771, 2004.

[12] M. Boudaoud, Y. Haddab, and Y. Le Gorrec, "Modeling and optimal force control of a nonlinear electrostatic microgripper," Mechatronics, IEEE/ASME Transactions on, vol. PP, no. 99, pp. 1 -10, 2012.

[13] A. Macchelli and C. Melchiorri, "Modeling and control of the Timoshenko beam. the distributed port Hamiltonian approach," SIAM Journal on Control and Optimization, vol. 43, no. 2, pp. 743-767, 2004.
[14] Y. Le Gorrec, H. Zwart, and B. Maschke, "Dirac structures and boundary control systems associated with skew-symmetric differential operators," SIAM Journal on Control and Optimization, vol. 44, no. 5, pp. 1864-1892, 2005.

[15] J. A. Villegas, "A port-Hamiltonian approach to distributed parameter systems," Ph.D. dissertation, Universiteit Twente, 2007.

[16] J. Villegas, H. Zwart, Y. Le Gorrec, and B. Maschke, "Exponential stability of a class of boundary control systems," IEEE Transactions on Automatic Control, vol. 54, pp. 142-147, 2009.

[17] B. Hamroun, L. Lefèvre, and E. Mendes, "Passivity based control of a reduced port-controlled Hamiltonian model for the shallow water equations," in Proceedings of the 47th IEEE Conference on Decision and Control. Cancun, Mexique: IEEE, 2008, pp. pp. 3917-3922.

[18] A. Baaiu, F. Couenne, L. Lefevre, Y. L. Gorrec, and M. Tayakout, "Structure-preserving infinite dimensional model reduction: Application to adsorption processes," Journal of Process Control, vol. 19, no. 3, pp. 394 - 404, 2009. [Online]. Available: http://www.sciencedirect.com/science/article/pii/S0959152408001212

[19] R. Moulla, L. Lefevre, and B. Maschke, "Geometric pseudospectral method for spatial integration of dynamical systems," Mathematical and Computer Modelling of Dynamical Systems, vol. 17, no. 1, pp. 85-104, 2011.

[20] R. Moulla, L. Lefvre, and B. Maschke, "Pseudo-spectral methods for the spatial symplectic reduction of open systems of conservation laws," Journal of Computational Physics, vol. 231, no. 4, pp. 1272 - 1292, 2012. [Online]. Available: http://www.sciencedirect.com/science/article/pii/S0021999111006012

[21] A. Macchelli, S. Stramigioli, and C. Melchiorri, "Port-based finite element model of a flexible link," in Proceedings of the 7th IFAC Symposium on Nonlinear Control Systems (NOLCOS'07), Pretoria, South Africa, 2007

[22] V. I. Arnold, Mathematical methods of classical mechanics. SpringerVerlag, New York, 1989.

[23] A. J. van der Schaft, L2-Gain and Passivity Techniques in Nonlinear Control, 2nd ed. New York, USA: Springer-Verlag, 2000. 